\title{
Kulturanthropologie als Veränderungswissenschaft ${ }^{1}$
}

\section{Manfred Faßler †}

\section{Zusammenfassung}

Es sagt sich so leicht: unsere Welt verändert sich. Was aber verändert sich oder wird verändert? Wodurch, durch wen, warum? Cui bono? Leben wir in einer Welt „kreativer Zufälle“, von denen der Physiker Klaus Mainzer (2007) spricht? Oder lenkt dies vom „Egoismus der Gene“ ab, den der britische Biologe Richard Dawkins (1976) betont. Geschieht oder passiert Veränderung? Wird sie gemacht? Sind ,Egoismen der Kultur', des Marktes, der Ökonomie daran beteiligt? Woher kommen diese? Welchen Sinn haben sie bei globalen Kooperations- und Konsensanforderungen? Und was ist zu tun, wenn sich herausstellt, dass die Bedingungen umwälzender Veränderungen menschengemacht und brutal und gewaltförmig sind, wie Saskia Sassen in ihrem Buch "Ausgrenzungen" (2015) beschrieb. Oder, wenn wir uns selbst, unsere Körper, unsere Seinsweisen, unsere Denkweisen absichtlich verändern, wir eine Cyborgisierung (Haraway 1991) betreiben? Muss Wissenschaft dann explizit politisch werden? Sollten die ersten Schritte eine „experimental respecification of sociality with digital technologies“ (Marres \& Gerlitz 2019, 3, Anm.1) sein? Mit diesen wenigen Fragen stelle ich verändern und machen in den Vordergrund meines Essays. Der Text ist ein Plädoyer für eine Kulturanthropologie, die sich dem reproduktiven Gattungsverhalten ebenso widmet wie Kultur-, Sozial-, Kommunikations-, Urbanisierungs-praxen, Kreativität, Maschinen- und Technologieentwicklungen.

Schlagwörter: Digitale Transformationen; 4. Industrie-kulturelle Revolution; Soziale Netzwerke; Kulturentstehung; new socials; Krise von Zusammenhängen; neue Kooperations-, Konflikt- und Konsensformate

Manfred Faßler †, Goethe-Universität, Institut für Europäische Ethnologie und Kulturanthropologie, Frankfurt am Main, Deutschland

\footnotetext{
${ }^{1}$ Anmerkung der Redaktion: Kurz nachdem Manfred Faßler diesen Text für die vorliegende Ausgabe der Kulturanthropologie Notizen fertiggestellt hatte, verstarb er völlig unerwartet am 17. April 2021. Wir danken seiner Familie, dass wir seinen letzten Text in diesem Band veröffentlichen dürfen. Er gab uns damit in der ihm unnachahmlichen Weise viele wichtige Gedanken und Aufforderungen mit auf den Weg, den wir nun ohne ihn weitergehen müssen. Seine ihm eigene Textgestaltung in Form von zahlreichen Unterpunkten, Aufzählungen und kurzen Absätzen haben wir weitestgehend erhalten, nur fehlende Referenzen ergänzt und offensichtliche Fehler korrigiert.
} 


\section{Annäherungen}

Dieser Text ist Versuch und Vorschlag zugleich. Versuch, weil eine Veränderungsforschung fehlt, auf deren sichere Begrifflichkeit ich mich beziehen könnte. Und Vorschlag, weil für Forschungs- und Theorieansätze noch einige Zeit mit Datennetzwerken, mit neuen Formen von Kulturalität und Sozialität „experimentiert" wird und werden muss, wie Noortje Marres und Carolin Gerlitz (2019) beschrieben.

Lebensverhältnisse, Kulturen und soziale Systeme verändern sich ständig. Der bekannte Orts-, Berufs- oder Tapetenwechsel verändert individuelle Verhältnisse wohl rascher als soziale Systeme verändert werden. Und Kulturen sind wohl die ,langsamsten' von den dreien, gefestigt durch übergenerationale, religiöse, gruppenspezifische oder regionale Normpflege. Alle drei stehen derzeit unter erheblichem Druck digitaler Erschließung der Welt oder weniger pathetisch: durch die Erschließung von Gesellschafts-, Kultur- und Wissensräume als digitale Geschäftsfelder. Nicht nur eine Infrastruktur der technologischen Konnektivität verfestigt sich durch globales Nutzer:innen-Verhalten. Es bildet sich eine (global differenzierende) "Culture of Connectivity" (van Dijk 2013) aus.

Unterstützt wird die globale Informationsindustrie dabei von dem Umbau bisheriger Produktions-, Berufs-, Kommunikations- und Kooperationsregeln. Umbau meint: Alle angesprochenen Regelbereiche werden in Programme übersetzt, patentiert, mit Copyrights versehen und zur Vermietung auf dem Weltmarkt der Ideen und Realitäten angeboten. Eine weitreichende Enteignung früheren sozialen, institutionellen, kreativen, gestalterischen Wissens geschieht. Und zugleich entstehen neue, nicht mehr territorial zuzuordnende digitale, datenbetriebene Netzwerke. Deren Marketing wird über Versprechen von enhancement, augmentation, elaboration betrieben. An einer Empirie und Theorie zu social augmentation mangelt es noch, es sei denn, man akzeptiert „Programmed Sociality“ nach Facebook- oder GoogleSetzungen (Bucher 2013). Michel Callon fragte 2006 zu Recht: „Can methods for analysing large numbers organize a productive dialogue with the actors they study?" (Callon 2006).

Am Beginn des 21. Jahrhunderts zeigen sich Umrisse einer (daten-, medien-, informations-) technologischen Zivilisation, in der nicht nur das „Außen des Menschen“ (Löffler 2019: 15ff.) das Lebensgeschehen massiv verändert. Konzepte von Individualität, Teilen und Teilhabe, von lebenslangem Lernen und lebenslang erforderlicher Kreativität, Subjekt und Solidarität, und Hirnforschungen ${ }^{2}$, um nur wenige Dimensionen hier anzusprechen, werden in den techno-sozialen Netzwerken unter den Verbraucher:innenmodellen Consumer, Prod-User oder User neu verfasst.

Ungewiss ist, wie die intermediären Zusammenhänge von Regeln, Konventionen, Normen und Gesetzen morgen aussehen werden. Deutlich wird, dass massive globale Entwicklungen hin zu global verteilten Bereichs-Monopolen, wie Amazon, Facebook, Google, TenCent, Huawei, nicht auf irgendeinem Gerätepark aufbauen. Wissen, Unwissen, Arbeits- und Familiensituationen, kommunikative Fähigkeiten, kognitive Ressourcen, soziale und kulturelle Konventionen von Menschen tragen diese Verbreitungsformen der Cybertechnologien mit.

Der Vorschlag, über Veränderungswissenschaft nachzudenken, nimmt den Leitgedanken von Serge Moscovici (1990) auf, dass auch Technologie zur "menschlichen Geschichte der

\footnotetext{
${ }^{2}$ Hinweisen möchte ich auf die Arbeiten am Institut für Kulturanthropologie und Europäische Ethnologie der Goethe Universität, vor allem Deschauer et al. (2009) und Deschauer et al. (2014).
} 
Natur" gehört. Gerade bei Technologie ist es auch Kultur, die Kultur macht, und nicht vorrangig "Natur der Kultur“ (Mühlmann 2011). Die Datentechnologien sind in sich eine neue Organisation des Reproduktionsegoismus der Menschheit.

Milliarden Menschen, von Subjekten zu User:innen, Konsument:innen umbenannt, müssen binnen weniger Jahrzehnte neue Zusammenhänge, Verhältnisse, erfinden. Und sie lassen sich suchend, experimentell und ökonomisch gezwungen auf neue Maßstäbe für Genauigkeit, Beteiligung, Kollektivität, Sharing, Debugging, Cookies, Data-Farms ein.

Die Wortwahlen für diese Veränderungen sind unentschieden. Vom Computerzeitalter, von „,wirklichkeitsindustriellen Komplexen“ (Rheingold 1992), von ,Großer Transformation" (Polanyi 1979; 2017 [1973]), Zeitenwende oder "Achsenzeit“ (in Anlehnung an Jaspers 1949), sich computertechnologisch durchsetzenden „universale[n] Entwicklungsprinzipien“ (Löffler 2019: 35), „Pax Technica“ (Howard 2015) oder „4. Industrielle Revolution“ (Schwab 2019) wird gesprochen. Es ist viel los auf unserem Planeten.

Welche ökonomischen, technologischen, wissenschaftlichen oder markteigenen Treiber Veränderungen beeinflussen könnten, ist nicht leicht zu sagen. Wie diese Veränderungen ,kulturalisiert' werden, erst recht nicht. Ebenso schwierig ist die Beschreibung, wie sich soziale Verfassungen und kulturelle Konsensregeln verändern und als Regeln und Übereinkünfte gesetzt werden. Es sind die Menschen, die also das Außen (Löffler 2019) zu ihrem Innen machen, und neues Außen entwerfen.

Diese begrifflichen und methodischen Stolpersteine finden sich im selben Umfeld mit nicht unerheblichen Schwierigkeiten, technosoziale Kontexte fachlich beschreiben und erforschen zu können. Die Komplexität, von der so gerne alltäglich und in Konferenzen gesprochen wird, ist noch weitgehend unterbestimmt. Und dies gilt vor allem für die Zeit- und Sozialverhältnisse, für das ,entworfene Morgen' und die damit verbundene gewünschte, geforderte, erwartete Kontinuität. Zeit entwickelt sich unter diesen Anforderungen zu einer sprachlichen Leerstelle, solange keine (beruhigenden, sachlichen) Umgangsweisen mit Nano- und Femto-Sekunde (10-9/10-12 sec) und mit Echtzeitkommunikation (also 1 sec Informationsunterschiede) eingeübt sind.

Wir benötigen eine kulturanthropologische Zeitwissenschaft der digitalen sozialen Netzwerke. Es lässt sich, im Markt- und Machtgestus, leicht von zukunftsrelevanten gegenwärtigen Prozessen sprechen. Kulturanthropologie der Veränderung wird sich mit Zeit und Zukunft als Kategorien der Wahrnehmung und des Entwerfens befassen müssen. Oder mit Fragen:

Was sollen programm-intelligente Maschinen lernen, um lebensdienliche Vernetzungen zu ermöglichen?

Wieviel Warte-, Reflexions- Entscheidungszeit muss dem Menschen belassen werden?

Was darf eine moral machine, was eine decision machine ,wissen' und entscheiden?

Was dürfen Menschen über diese Maschinen, über ihre Betriebslogiken, ihre programmierten Aufträge wissen?

Und dies nicht nur gefragt in Richtung autonom fahrender Autos, sondern in Richtung social/super scoring (Gapski \& Packard 2021), künstlich intelligente Öffentlichkeiten, smart cities. Und: wie bilden sich Subjektivität, Personalität, Wissen, Kooperation, Rollenstrukturen, Körperlichkeit aus unter Bedingungen von virtuellen Tele-Welten? Fachlich hilfreich könnte dafür der Praxis-Ansatz sein, der mit dem Konzept der Science Technology Studies (STS) (siehe Laboratory: Anthropology of Environment I Human Relations 2019) verbunden ist. 
Mit wenigen Beispielen werde ich versuchen, mich der Machart und der Machtart gegenwärtiger technologischer, sozialer, kommunikativer, normativer Veränderungen zu nähern. Die Datenökonomien, die ich kulturanthropologisch berücksichtige, bilden Netzwerke globaler "Systeme der Umverteilung“ von Macht (Popitz 1992: 218ff.), und auch ihrer Reproduktion, ob als Informationsgesellschaft, Lebenslangen Lernens, Künstlicher Intelligenz oder Computerspiele. Die damit verbundene Vielfalt der Wechselwirkungen von Menschen, Maschinen, Technologien, Medien, Wohn- und Lebensweisen, Orten und Globalität werde ich nicht in diesem kurzen Text darlegen können.

Wichtig ist mir zu betonen, dass mit den digitalen Technologien nicht Maschinen-Parks gemeint sind, sondern Denk-, Organisations-, Verständigungs-, Entwurfs-, Koordinations-, Werbe-, Spiel-, Überwachungs- und Kontrollpraktiken.

Und diese Praktiken bringen soziale Zusatzräume hervor, die von Territorien, Gesellschaften nicht nur abgelöst sind. Sie schwächen deren Bedeutung durch vorläuferlose Vernetzungen, Varianten von Abhängigkeit, durch gigantische Märkte und Logistiken.

Datenökonomische Ereignishorizonte lassen ahnen, dass nicht nur Politik, Technologie und Ökonomie von der Zustimmung der Bürger:innen entkoppelt und neu verfasst werden. Überlieferte kulturelle, rechtliche, wissensökonomische Zusammenhänge werden, so lässt sich vermuten, ihre gesellschaftlichen Integrationsfunktionen zugunsten globaler, netztechnisch verbundener Kurzzeitsozialitäten einbüßen.

Kulturanthropologie könnte sich sehr rasch zeitlich, technologisch und ökonomisch instabilen Netz-Bevölkerungen und Moment-Kulturen gegenübersehen. Sie wird sich nicht nur mit einzelnen Veränderungen befassen müssen, sondern mit generativer Veränderung. Eine Schlussfolgerung ist, sich nicht stabiler Gemeinschaften oder Kulturen zu versichern, sondern eine Kulturanthropologie der Transformation, Konflikte, Kooperation, des Konsens, der Veränderungs-Praktiken zu entwerfen. Nun, es gibt sie noch nicht. Dies legt nahe, nicht entwerfend übermütig zu werden, sondern vom Versuch zu sprechen.

Für die Rückbezüge auf anthropologische Theorie und Forschungen habe ich unter anderen den Anthropologen Gregory Bateson (1996 [1985]; 1997 [1987]) und den Naturwissenschaftler Heinz von Foerster (2008) zu Rate gezogen. Ohne sie ständig zu zitieren, lieferten ihre Arbeiten beeindruckende und hilfreiche Beispiele für eine systemische Anthropologie. Sie sind für eine heutige Neufassung von kulturanthropologischer Forschung - aus meiner Sicht - sehr wertvoll.

\section{Unterschiede und Muster}

Der Politikwissenschaftler und Soziologe Colin Crouch notierte 2015 in seinem Buch über die Neoliberalisierung der Wissensbestände, die er als Bedrohung des „lebensnotwendigen Wissens" ansieht:

„Unsere Abhängigkeit vom Wissen verwandelt sich auf diese Weise schleichend in eine Abhängigkeit von solchen Vertretern privater Interessen, deren Verhalten sich lediglich in dem Maße an Moral und Ethik orientiert, in dem sie vom Markt dazu gezwungen werden - oder eben nicht." (Crouch 2015: 103)

Märkten gehorchend, hätten schon mehr Menschen von der „Fälschung von Informationen“, vom Baum des [...] Bösen“ (ebd.: 239) gegessen, als von der „Erkenntnis des Guten“. Dieses 
alte Wortspiel benennt Aktuelles: der Kontext und die Wahrheitsbedingungen von wissenschaftlichem Wissen werden derzeit nicht nur angezweifelt, - und dies nicht erst seit Donald Trump oder Margret Thatcher. In aktivierten Modellen des Maschinen-Denkens, -Entscheidens und -Lernens wird nicht nur das multisensorische Vermögen des Menschen verkümmert dargestellt. Die evolutionäre Anforderung des Denkens, der Wahrnehmung, der Kreativität, sich gegenüber anderem Denken, anderer Wahrnehmung, anderer menschlicher Kreativität zu beweisen, zu bekräftigen, wird banalisiert. Datenökonomie, hier verstanden als selektive, marktspezifische Anwendung der Datentechnologie, und Neoliberalismus treten gleichzeitig auf. Datenökonomie und Neoliberalismus verbindet die (be-)deutungsfeindliche „Fälschung von Informationen“ (Crouch 2015).

Es sind Bestrebungen, sich völlig von den bisher entstandenen Sozialen Strukturen und Gruppierungen zu lösen, die produktive Differenzierung von Kulturen, Ethnien, Sozialen Verbänden, Sozialen Systemen, Wissensentwicklungen zu verlassen und nur auf ein (echtzeitiges) Reaktionsgeschehen zu setzen, auf formlose, entscheidungslose Daten.

Aber diese gibt es nicht. Jedes Datum setzt Unterscheidung und Entscheidung voraus, gerade dann, wenn Daten zu Informationen werden, also Bedeutungssteuerung durchsetzen. Sich auf Daten als formlose Unterscheidung zu berufen „is an evolutionary failing of the human brain" (du Sautoy 2019: 91). Sich auf den Consumer/User als einzige relevante formlose Unterscheidung zu berufen, ist eine Fehlentwicklung menschlicher Sozialverhältnisse, - obzwar machtpolitisch nachvollziehbar.

Marcus du Sautoy stellt in „The Creativity Code“ heraus:

"The lesson: the machine may be learning but you need to make sure it's learning the right thing. This is becoming an increasingly important issue as algorithms trained on data begin to affect society. Mortgage applications, policing decisions and health advice are being increasingly produced by algorithms." (ebd.: 97)

Kein Datenstatus, kein errechneter Zustand ist grund- und bedingungslos.

Keine Differenz, ob gegenständlich, sozial, kulturell, modisch, ästhetisch, oder als Schicht, Klasse, Community, ist voraussetzungslos. Es geht mir also um Voraussetzungen, Werden und Verschwinden von Formen, Zusammenhänge, Entscheidungen, kritische Konstellationen. Das alles schwebt im Hintergrund mit bei der Frage:

Welche inhärenten Wirkungen erreichen welche Lebensverhältnisse (und Dinge)?

Woher kommt die Angst vor Komplexität? (Vester 2019: 13-97)

Und welche enorm unterschiedlichen ,Aufgaben' kommen auf Kontinuitätsträger wie Maschinenstrukturen, Ökonomie, Ethnien, Institutionen, Verhaltenskodierungen, Schulsysteme, Wissenssysteme und Subjekt / Person zu?

Die wenigen, über Colin Crouch, Marcus du Sautoy und Frederic Vester, angedeuteten Hinweise kündigen Forderungen an neue Bedingungen, Unterscheidungen, Formen sozialer Selbstorganisation an. Dabei sind die Entstehungsbedingungen von Ethnos, Demos, Kultur nicht denen von Maschinen, Programmen, Institutionen gleichgestellt. Dennoch: Dort, wo sie zusammentreffen, in den spezifischen Vernetzungen, in Wirkungszusammenhängen, haben sie viel miteinander ,zu tun'. Und es wird mehr werden, je enger die Vernetzungen werden. 


\section{Digitale Maßanzüge, Konsumentenvölker, Deprogrammierung des Gesellschaftlichen}

Auch wenn das Marketingversprechen der "sozialen Netzwerke“ und die Milliarden User:innen diese als ,soziales' Regelwerk durchsetzen können, sollten wir wissenschaftlich und politisch-emanzipatorisch genau auf das real-time using sehen.

Nochmals kurz Marcus du Sautoy:

"There have been calls for the industry to try to develop a meta-language that the algorithm can use to justify its choices, but until this is successfully done we may have to be more cautious about the impact of these algortihms in everyday life" (du Sautoy 2019: 95)

Um dies nicht falsch zu verstehen: Das Vorhaben, die digitalen Welten zu erzeugen und fortzusetzen, - von Individualdaten, über Gruppen- und Verbunddaten, zu Produktionsdaten, computertechnischen Entwicklung von Bakterien und Viren, 3D-Druckerei von Häuserwänden, Schmuckstücken und künstlichen Herzen - ist sozial, ökonomisch, wissensgeschichtlich unumkehrbar. Und dennoch ist deren Programmierung und Gestaltung in Menschenhand. Es sind keine finalen Prozesse; sie sind in und durch ihre Milliarden Netzwerke in Computern und zwischen ihnen im ernsten Sinne ziellos.

Über Jahrtausende sind die Systeme der Zahligkeit (Numerosität), der Schriftzeichen, ihrer grammatischen Abstraktionen, der Mechanisierung und Maschinisierung, des Abacus, der Rechenmechaniken, bis zu Computern erfunden und angewandt worden. Der französische Anthropologe André Leroi-Gourhan hat in seinem Werk „Hand und Wort. Die Evolution von Technik, Sprache und Kunst“ (1980) diese Zusammenhänge ebenso wunderbar dargestellt, wie dies Serge Moscovici in seinem „Versuch über die menschliche Geschichte der Natur" (1982) und Edward O. Wilson in "Die Einheit des Wissens" (20013) tat.

Alle diese Arbeiten zeigen die inter- und transkulturellen Anpassungs- und Lernverläufe hilfreicher Modelle, Konventionen und Abstraktionen auf.

Dabei ist für die Computerdiskussion sicherlich wichtig, wie sich die "biography of a dangerous idea“ weltweit gestaltete. Charles Seife (2000) meinte damit "ZERO“, also die hilfreiche Erfindung der NULL im Hindus-Tal. Dazu gehört auch die Trennung der modernen Mathematik von der Philosophie (Peckhaus 2005), sowie die Eingrenzung bestimmter mathematischer Gesetze auf „Die Herrschaft der Regel“, wie die sehr lesenswerte "Grundlagengeschichte des Computers" von Bettina Heintz (1993) titelt.

Die abstrakten Systeme gehören also in ihrer Erfindungs- und Anwendungsgeschichte zum Menschen und lassen sich als globale Lern- und Praxisverläufe darstellen. Und sie werden für immer mehr Bereiche sozialer Selbstorganisation bedeutend.

Gerade deshalb steht das Ringen um Vorstellungen des Sozialen, um deren Entwurf und Entwicklungen an, ebenso die neuerliche Kontroverse um Leben, Ästhetik, Architektur, von Gruppenzusammenhängen und -Kontinuität. Weder Gruppen, noch ,Soziales', weder Demos noch Ethnos sind ,externe Effekte' der Datenprogramme. Sie sind deren intelligente, affektive, unterscheidungsfähige Grundlage menschlicher Kooperation und Koaktionalität - und zwar als (instabile) Praxis der Menschen, nicht als irgendeine Wesensbestimmung von Menschengruppen. 
Gerade auch wegen der gigantischen Schatten der Abstraktionsverläufe sind Veränderungen nicht einfach, aber, wie die Entwicklungsgeschichten zeigen: sie sind machbar. Auswege aus der Massendaten-Realität sind: Sinne, Wahrnehmung, Aufmerksamkeit, Reflexivität, und Konsens gegen die aggressiven Zeitökonomien, die Reduktion von Subjektivität auf User:innen-Dasein etc. zu stellen. Dies kann nur gelingen, wenn offengelegt wird, welche Organisations- und Ordnungsimperative in den Unterschieden angelegt sind, die digitalisiert werden.

Denn: „Numbers never start from a tabula rasa, but from a prefiguration of difference“ (du Sautoy 2020: 68).

Beschränken wir uns auf den Menschen als ,user', trifft die Feststellung:

"This is an evolutionary failing of the human brain" (du Sautoy 2020: 91).

In der viel zu wenig diskutierten und erforschten Dauer-Verbindung von User und Social Networks herrscht die Bit-Orientierung vor, die Datenmassen und Echtzeit. Begleitet ist diese von multisensorischer Körperorientierung, die Daten über Augenbewegung, Schreibkommunikation, Farbwahl, Schreibgeschwindigkeit ,absaugt'. Auch die schrift- und bildkulturellen Dimensionen von Verständigung, also die indirekte Kommunikation, werden ins Digitale der Plattform-Globalität der Big Nine (Webb 2019) und der Monostruktur $>$ User \& Social Network< zerlegt.

Aber:

$\mathrm{Zu}$ welchen sozialen Großgefügen gehören ,social networks'?

Stehen sie für einen bislang nicht empirisch unterlegten Trend zur "Weltgesellschaft" (Luhmann 1984)?

Oder sind die Ordnungsgiganten der Netzwerk- und Plattform-Kapitale (sei es Amazon, Google, GPS, Galileo, Alibaba, TenCent, etc.) an die Stelle von Gesellschaften, Demos, Ethnos, regionaler Kultur getreten?

Bleibt von der geografischen ,Region` nur noch der Gemüseanbau, zelebriert bei EDEKA, Rewe, Lidl., während sozio-kulturelle und soziale Präsenz in ,social networks' beheimatet werden?

Bilden sich in den fortwährend erweiterten Verbindungen, in der sogenannten Algorithmisierung von ,social network' eine Systematik nach-territorialer (topologischer) vorläufiger Kulturen und Sozialverhältnisse aus?

Möglich ist's.

Umso mehr müssen sich Wissenschaften um eine Art (begriffliches, empirisches, konzeptionells) re-framing kümmern, um die Erforschung von Online-Offline-Populationen, deren Umgang mit sich selbst, deren Kooperations- und Kollaborationsfähigkeiten. Damit verbunden sind Anforderungen an Forschungs- und Theorieentwürfe, die nicht der (kausalen, linearen) Funktionalität folgen, sondern Wechselwirkungen. Diese lassen sich als Bedingungen „maximalen Stresses“ (Mühlmann 2011) oder als Zustand ko-evolutionärer Stressregulierung (Faßler 2009; 2014) erfassen.

Wichtig ist mir die Feststellung:

Kein Daten-Paket, ganz gleich welcher Komplexität, führt zu lebensdienlichen Zusammenhängen oder Erklärungen. Das Soziale des Homo sapiens ist keine genetisch determinierte Praxis, kein technologisch determinierter Befehlsraum, ist nicht dem Termitenbau 
oder einer Ameisenkolonie vergleichbar. Strukturen, Dinge, Verständigung, Absicht, Vorausdenken, Anpassen, unterscheiden und entscheiden und etliches mehr, werden herangezogen werden müssen, um die Kapazitäten des Sozialen und Kulturellen grundlegend und in dem Daten-Universum zu verstehen.

Kein Datenereignis ist automatisch Teil eines Maschinengefüges. Es sind in weitem Sinne sozio-kulturelle, techno-ökonomische Dinge, die dazu beitragen. Wir müssen uns schon um deren Verbindungen bemühen, sie deuten, erklären, fördern oder verhindern.

Die aktuell werbend geforderte ,Digitalisierung' der Schulen, Universitäten, Kindergärten, Wohnungen (Home-Offices) sollte mit den Fragen verbunden werden:

Welches Lernen, welches Wissen, welches Wohnen, welches Vertrauen wollen wir?

Und welche Gruppen bemühen sich um welches Wissen etc.?

Welche Beiträge können Wissenschaften und Wissenschaftler:innen leisten.

Und: Was sollen die fortschreitend lernfähigen Algorithmen lernen? Zumal sie ja auch in Schulen eingesetzt werden (sollen).

Das alles ist hier nicht zu beantworten. Der Versuch eines kleinen Überblickes lohnt sich aber.

\section{„End of“" oder: Sorge um die begriffliche Ordnung?}

Veränderung lässt sich als offene, anpassungssensitive Entwicklung denken, unscharfen Gesetzen (Riedl 2000; 2003) der Kooperation eigen. Das heißt: Es gibt für keinen ,sozialen Zustand“ irgendeine normative Mengenvorgabe, weder für Bevölkerung, Berufsgruppen, noch für Klassen, Schichten. Vielleicht für die Zahl von Bandmitgliedern bei Boy-Groups oder Tanzwettbewerben, Fußballmannschaften - nun, darum geht es mir hier nicht.

Die Menschheitsgeschichte, damit auch unsere vielfältige Gegenwart, hat nicht nur

Energie (Feuer/Wärme/Architektur/Mobilität/Bekleidung/Wohnen),

Metabolismus (Ernährung, Jagd, Sesshaftigkeit, Kunstdünger, Nahrungsindustrie, Hunger)

und

Informationen (multisensorischer Körper, Nachricht, Wissen, Experiment, Handwerk, Bildlichkeit, Numerosität)

im Gepäck. ${ }^{3}$

Zur Geschichte gehören vielfältige Formate des Sozialen, der Organisation als Sippe, Familie, Dynastie, Dorf, Stadt, Reich, Staat, Diktatur, Demokratie, als Kollegialität, Genossenschaft, Nachbarschaft, Chor, Gemeinde, Orchester, Rockgruppe. Übergänge zwischen alldem können individuell, kollektiv, verfahrensgerecht, partizipatorisch ,geschafft' werden, durch klare Zeitabsprachen, Verabredungen, Konzertbeginne. In ihrer Verallgemeinerung als Bestandteile komplexer Sozialsysteme bleiben Momente der Entstehung, Vielfalt beteiligter Ideen und deren Wechselwirkungen unscharf.

\footnotetext{
${ }^{3}$ Siehe auch: Entwurf des Energie-Metabolismus-Inforation/EMI-Models in Faßler 2009: 287-291.
} 
Anthropologie und Soziologie, die ich hier in die Waagschale lege, müssen auf die evolutionäre ,Unschärfe' (von Foerster 2008; Iser 2013) ihrer Arbeitsbegriffe nicht nur vorbereitet sein und reagieren können. Sie sollten ihre Begriffs- und Forschungsregeln in Vorläufigkeit, in "context-sensitivity“ (Iser 2013: 193ff.) begründen.

Hart dagegen gerichtet war die Proklamation von Chris Anderson, führender Computerentwickler in den USA, am 23. Juni 2008. An diesem Tag titelte er: „The End of Theory: The Data Deluge Makes the Scientific Method Obsolete“ (Anderson 2008). Datenmassen, damals in Petabytes summiert, würden jede wissenschaftliche Bedeutungssuche sozialer oder individueller Prozesse überflüssig machen. Die sich selbst rechnenden Datenmengen zeigten: „All models are wrong, but some are useful“ (ebd.). Kausalität sei überflüssig und als Methode falsch. Neben der damals schon viel gepriesenen „Connectivity“ stand der Satz: „Correlation is enough". Daten, deren Programmsprachen eine Schaltung erlaubten, die sich also ,verstanden', sollten ausreichen, um soziale Zustände zu diskutieren. Dies ist Teil des Unsinns, nur Datenmassen könnten den Menschen bei der Lösung von existenziellen oder funktionalen Problemen helfen. Der Wirtschaftspsychologe Claus Triebel brachte dies am 02.07.2013 auf den Punkt: „Korrelation ist [...] die neue Kausalität“ (Triebel 2013).

Der Weg für Big Data wurde so gezeichnet (Mayer-Schönberger \& Cukier 2013). Vieles ist auf diesem Wege auch so gekommen: Entlastet von gesellschaftlichen Verantwortungsund Zusammenhangs-Diskursen baut die Big Data-Ökonomie (irgendwann Plattform-Ökonomie genannt) von Google, Facebook, Twitter, Amazon etc. eine Gegenwelt zu allem auf, was nicht digitalisiert wird, und zu allen Sozialverhältnissen, die bis zu diesem Zeitpunkt durch ethische, sozial-staatliche, solidarische Prüfungen legitimiert wurden. So steht hinter dem „End of Theory“ auch ein „End of Policy“, - und auch ein Eingang zu VerschwörungsIdeologien aller Art.

Obwohl ich biografisch den Computertechnologien der Jahre 1980-2000 nahestand, halte ich die völlige Abspaltung von Theorie, Normativität, sozialer Verantwortung, soziokultureller Wechselwirkung für bedrohlich.

Ich betone hier ausdrücklich den wissenschaftlichen Bedarf an geordneten Häufigkeiten, also Empirie. Und den Bedarf an Theorie. Die Zahlen, Korrelationen, Zusammenhangsmodelle und so weiter müssen methodisch überprüfbar sein. Gerade gegen wissenschaftliche Methoden richtet sich Chris Anderson (2008). Für die Kritik an den Mainstreams von Big Data, Social Scoring, Cambridge Analytica heißt dies: nicht Ende von Theorie, sondern Offenlegen der Programmierlogiken, der kulturellen Ordnungsimperative, der Pflichtenhefte, und (inzwischen) der monopolen Machtabsicht. Die monopolen Verwaltungen der Massen von (entkräfteten) Unterschieden führen dazu, dass die Ansprüche auf eine lebensdienliche, bis in jede Individualität hineinwirkende und garantierte Lebenswelt tendenziell verunglimpft werden.

Das „Ende der Theorie“ steht dann für das Ende politischer Legitimation oder auch für das Ende von Bedeutung. Hiergegen müssen sich die Fächer wappnen, über die ich hier schreibe. Zahlreiche Ansätze liegen vor, die sich dem Theorie-Abgesang von Anderson nicht unterwerfen. In allen geht es darum, die Bedeutungsvielfalt von sozialen Differenzen nicht nur zu ,managen', sondern in ihrer Wertigkeit für intelligente, kreative und demokratische Entwicklungen neu zu bestimmen.

Auf wenige möchte ich hier nur hinweisen. So Stefania Milan (2015) „When Algorithms Shape Collective Action“, Emily van der Nagel (2018) „Networks that work too well“, Anne 
Helmond (2013) „The Algorithmization of the Hyperlink“, José van Dijck et al. (2019) „,Reframing platform power" oder auch Nick Couldry und Hendrik Jenkins (2014) „Participations: Dialogues on the Partcipatory Promise of Contemporary Culture and Politics“. Diese Arbeiten beruhen alle auf dem Gedanken, dass die technologische Durchdringung der Sozial-, Kultur-, Wissensbestände irreversibel ist. Und sie fordern neue Einsichten in die Entstehung, Art der Kontinuität, Entwicklung sozialer Zusammenhänge ein, indem sie sich gegen den ,Großen Abschied' von Theorie und Bedeutungskonflikten nach Anderson richten.

Die Suche nach den Bedingungen von Sozialität löst sich nicht nur von „Wired Magazin“, dem Publikations-Organ der Thesen von Anderson. Distanz lässt sich auch finden gegenüber den Strategie-Papieren von Google, Microsoft, Facebook - sofern öffentlich zugänglich. Die angesprochene ,Suche' schloss und schließt mit ein, nicht nur den Digital-Technologien gegenüber kritisch zu sein, sondern auch gegenüber den überlieferten Relationen zwischen Industrie, Bürokratie, Normen, Institutionen und Individuum, Subjekt, Gruppe, Kultur.

Das „stählerne Gehäuse“ der Schwerindustrie, von dem der Soziologe Max Weber zu Beginn des 20. Jahrhunderts sprach, rostet nicht nur; es wird zum Überbleibsel, zur Ordnungsruine. Tritt an seine Stelle die Organisationsmacht der Datenströme, der Echtzeitkommunikation, technogene Smart-Cultures, irgendwie/irgendwo verteilt in den Netzwerken der Welt? Nortje Marres und Carolin Gerlitz (2019) brachten die Situation auf den Punkt: „On the experimentalization of sociality in digital environments, detecting sociality with social media“. Beides: Entwicklung und Experiment zeigen auf, dass es keine endgültigen Synthesen des Menschen mit egal welcher Umwelt gibt. Damit liegt Verantwortung für die Art der Eingriffe bei uns Menschen. Und einen Verantwortungsbereich bilden Wissenschaften.

\section{Kultur, unabsichtlich / absichtlich}

Welche Formen der sozialen und kulturellen Selbstorganisation werden uns Menschen unter den Bedingungen der globalen „Vierten Industriellen Revolution“ (Schwab 2019), kurz: „Industrie $4.0^{\prime \prime}$, begleiten?

Schaut man genauer hin, ist die Frage ein Schwergewicht.

Denn sie legt offen, dass wir uns wissenschaftlich damit sehr schwertun, Entstehung, Dauer, kognitive Wirkungen, soziale und kulturelle Verbund- und Zusammenhangslogiken von den Formationen zu erklären, die wir Kultur oder Soziales nennen.

Deren Entstehung, Wirkungsweisen, Veränderungen sind multiple Prozesse. In ihnen (und an ihnen) sind u.a. Naturgesetze, Gesetze des "extended phenotype“ (Dawkins 1982), des expressiven, entwerfenden, gestaltenden menschlichen Lebens, der Produktion, der Macht, der Sesshaftigkeit, komplexer Lernverläufe, ortsbildende Hierarchien, Mobilität, politische und rechtliche Verfassungen ,aktiv' beteiligt. Ändert sich diese (nicht-intentionale) Gesetzes-Bindung, wenn man auf absichtlich ,gestaltete', vererbte, , vererbbare', ,gepflegte Lebensweisen deutet, um die es bei Ethnos, Demos, Kultur, Identität, Subjekt geht? Keineswegs.

Die Frage, welche Sozialformen uns begleiten werden, führt, wie alle genannten Wörter, nicht in vermeintlich zufällig entstehende Verhältnisse. Schon gar nicht heute und in den kommenden Jahrzehnten. Wenn Microsoft, Amazon, Google, Facebook und IBM 2016 eine „KI-Partnerschaft ins Leben“ rufen mit dem Ziel, „Bewusstsein für KI zu fördern, ...bewährte Praktiken zu den Herausforderungen und Chancen auf diesem Gebiet zu formulieren“, so Satya Nadella, CEO von Microsoft (zitiert nach Schwab 2019: 7) so geht es um „Zu- 
gang zu Informationen demokratisieren“ (ebd.: 8), um „standortunabhängige“ Demokratisierung (ebd.: 9), darum, „das regulatorische Umfeld“ eben standortunabhängig zu „revitalisieren“ (ebd.: 9). Demos, Ethnos, Kultur, Vertrauen werden als Produkte erkennbar, - fern der Idee irgendeiner Essentialität. Technologie als grundlegende Dimension der Sozialverfassung wird nicht angesprochen.

Diesen strategischen Muskelspielereien von Satya Nadella steht entgegen: Auch Microsoft oder Baidu, auch Amazon oder TenCent, können nur Naturgesetze und über lange Entwicklungsverläufe entstandene kognitive Fähigkeiten anwenden.

Mit Natur zu verhandeln, gelingt auch ihnen nicht. Allerdings gelingen ihnen massive Eingriffe in die menschengemachten Kommunikationswelten, in visuelle Anschauung, räumliche, zeitliche, örtliche Wahrnehmung, Denkgewohnheiten, Sozialkonzepte. Mit ihren globalen User:innen-Völkern schaffen sie den Abschied von der schwerindustriellen und bürokratischen Moderne, im Gewand ihrer Produkte: Fernsehen, Telefonnetz, Automobilität, Überwachung, Social Networks. So entstehen machtpolitische Positionen durch virtuelle Realitäten, Second Life-Angebote, Online-Sozialität, Globale Community-Märkte (Social Networks genannt) etc. Sie beeinflussen Entstehung und Erhalt von Sozialsystemen, legen die Variationsbreiten von Kultur, Wissen, Gestaltung, Infrastruktur, Transport- und Speicherlogistik fest.

Aber es ist nicht nur ihre Funktionsmacht, die kritisch zu betrachten ist. Es ist auch die Ignoranz gegenüber vernetzten Naturgesetzen und das falsch gedachte Verhältnis von Technologie/Produktion/Ökonomie und von im weiten Sinne Kultur. Dem angedeuteten Machtgestus entstammt das Modell, Soziales und Kultur seien "externe Effekte“ (so mehrmals bei Schwab (2019) zu lesen), oder, nochmals Nadella (zitiert nach Schwab 2019: 7): „Hinter jeder Erfahrung steht Künstliche Intelligenz, die unsere menschlichen Fähigkeiten um Erkenntnisse und Prognosekräfte erweitert."

Ich gehe von einem umgekehrten Verhältnis aus. Hinter jeder Variante „künstlicher Intelligenz" stehen komplex zusammengesetztes Wissen und Entscheidungen über deren Anwendungsbereiche:

Kultur und Soziales sind keine „externen Effekte“.

Sie sind für menschliches Leben konstitutiv, auch für Ökonomie und Künstliche Intelligenz.

Geht man von der ,Vorherrschaft' des Digitalen aus, wirft dies die Frage auf: Wie lässt sich Kultur und Soziales formulieren, wenn das Prinzip ökonomischer Reproduktion dasselbe ist, wie das sozialer Kommunikation, der Wissensspeicherung, der Spiele, des Entwerfens, der Verabredungen: eben digital? Erodieren die klassischen Moderne-Unterscheidungen, die seit circa 200 Jahren das Phänomen des ,Bürgerlichen' profilieren? Die Fragen an die gegenwärtigen Transformationsverläufe müssten lauten:

Wieviel Gesellschaft, wieviel souveräne Kultur verträgt globale Technologie?

Wieviel Demokratie verträgt sie?

Wieviel und welche Form von Ethnos verträgt globale Künstliche Intelligenz, globale Netztechnologie, Geoökonomie?

Können diese Kategorien noch als Referenten für Volks- oder Kultur(en)souveränität aufgerufen werden? 


\section{Kurzgefasst:}

Wir wissen, wie ein Algorithmus arbeitet. Ist er als selbstlernender Automat konzipiert, wissen wir nicht, wohin sich die Schaltungsinhalte entwickeln. Sollten Menschen nur von den Ergebnissen lernen, deren Zustandsordnungen adaptieren, damit umgehen, werden sie die Gründe und die weiteren Folgen dieser lernenden/entscheidenden/moralischen Maschinen auch nicht kennen lernen.

Aus meiner Sicht mangelt es an einer Kulturanthropologie der Abstraktion.

Es reicht nicht, sich Archäologien und Archiven zu widmen, sich auf Tradierungen und Traditionen zu konzentrieren. Deren Erfolg für das Verständnis der Herkunft von Gruppen, Verhalten, Moden, Architekturen, spezifischen Repräsentationen steht damit nicht in Abrede. Es geht mir dabei eher um die Erforschung von entstandenen, gemachten Differenzen und den Entscheidungsgründen, einen spezifischen Weg zu normieren, zu ordnen, zur offiziellen Ordnung zu machen, zu repräsentieren und zum Referenzsystem zu erhöhen. Erst, wenn wir diese Prozesse als Modelle entwickelt haben, werden wir auch auf die Komplexität von erdachten Datenwelten, Datenordnungen, von entworfenen Wegen, Daten als relevante Informationen zu verwenden, Einfluss nehmen können.

Erforderlich ist

Wissen um die verallgemeinerten Abstraktionen, mit denen wir Menschen unser konkretes Leben gestalten, und

Wissen um die Praxen, die die Gründe der digitalen Transformationen offenlegen können, um sie zu verändern.

Dies ließe sich als Kulturanthropologie der Infrastrukturen, der (soziotechnischen, kybernetischen) Netzwerkbildung, ausweiten.

Bleiben wir bei der Frage, mit welchen Mustern die 5 Milliarden User:innen ihre Verbindungen pflegen, mit welchen sozialen, ethischen, humanitären, demokratischen Normen sie jeden Tag ihre Accounts öffnen und bedienen. Wenn die beiden Google-Vordenker Eric Schmidt und Jared Cohen und ihr Büchlein „The New Digital Age“ (2013) mit dem Satz eröffnen: „Soon everyone on earth will be connected“ und dies mit dem Versprechen verbinden: „We will offer You a kind of social contract“, stellt sich mir die (europäische) Frage: Wie sind die Motivlagen der User:innen? Wie stehen Offline-Realitäten zu Online-Realitäten? Können sie kollaborieren? Gibt es „patterns“ (Bateson 1996 [1985]) dafür? Oder lassen sich diese entwickeln? Gelten diese trans-kulturell? Definieren sie erdweite Gruppenzusammenhänge, die über die Berufstreffen im Flughafen, Stake-holder Konferenzen oder Flash-Mobs hinausgehen?

In diesen von uns Menschen erzeugten und belebten Realitäten entscheidet sich, in welcher Weise uns lebensdienliche Organisations- und Reproduktionsweisen entstehen. Oder aber, ob wir bei der Bewältigung von Lebensgeschick einknicken, langfristig versagen.

Umgekehrt heißt dies: keine einzige menschliche Lebensorganisation ist genetisch determiniert. Kein Hammer, keine Zisterne, keine Computermaus, kein Programm sogenannter künstlicher Intelligenz ist ,vorgesehen', keine Herrschaftsform lässt sich ohne Machtinteressen (Popitz 1992), bauen'.

Wir, der Mensch, sind unsere eigene generative Realität. 
Es gibt für diese Andeutungen etliche grundlagentheoretische, ordnende Begriffe, sei es Komplexität (von Foerster 2008), Emergenz (Iser 2013), Netzwerke (Riedl 2000), kreativer Zufall (Mainzer 2007), Autopoiesis (Maturana \& Varela 2015 [1990]), Kontingenz und Ironie (Rorty 1992) u.v.a.m. In allen sind zwei fordernde Empfehlungen enthalten:

Vermeide endgültige Aussagen, denn kein biografischer, sozialer, kultureller, kognitiver Lebens-Zustand ist Endzustand.

Und:

Hüte Dich vor Konzepten geschlossener Identität oder Wesentlichkeit, ob individuell, gruppenspezifisch oder sozialsystemisch.

\section{Ringen um Abstraktion und Anwendung}

Es gibt keine ,Eigenentwicklung' einer Maschine, keine ,natürliche` Begründung für irgendein Werkzeug, irgendeine Maschine oder Technologie. Der Mensch ist und bleibt der entwerfende, eingreifende Akteur (Moscovici 1990; Kelly 1997; Boyer 2002; Schurz 2011), bleibt ein umweltgebundenes Selbst. Technologien sind 100 Prozent menschengemacht. Soziale Selbstorganisationweisen ebenso (Wilson 2013).

Menschen ringen seit Jahrtausenden um und mit den Abstraktionen, die ihnen einfallen. Es sind die oft besprochenen bild-, zahlen-, schriftsprachlichen Systeme Zweiter Ordnung. Und sie ringen um die Praxis, um anpassendes Verhalten, um die Entzifferung ihrer Konkretion, um Anwendung, Verbreitung, Nutzungsbedingung der - im weiten Sinne - Techniken. Es ist dies immer ein Ringen um Wissen, Verfügungsrechte, Macht, um Reproduktionsverhältnisse und Lebensweisen. Und dieses Ringen nimmt die verschiedensten Formen des „Transfers von Information“ zwischen Menschen und Menschengruppen an, „unabhängig davon, ob dieser Transfer auf genetischen, epigenetischen oder phänotypischen Bahnen erfolgt" (Wieser 2007: 150f.). In den Transfers und durch sie entstehen Gruppen, Schichten, Hierarchien, Organisationsweisen, Legalisierungen. Somit lassen sich Forschungen zu jenen Gruppen, Ethnien, zu Demos und Anthropos nicht von den erfundenen Dingen, nicht von erfundenen Formaten der Selbstorganisation lösen.

Wir, Homo sapiens, kon-struieren und kon-figurieren. Wir ,klären uns auf' oder verdummen uns epochenweise, wir bauen, reißen ab, lassen verfallen, erfinden und nutzen Schriften, Zahlen, Formeln, Regeln, wir zimmern Aktenschränke und füllen diese mit gedanklicher und organisatorischer Lagerware, und sind stolz auf die abgeschlossene(n) Geschichte(n).

Es ist ein grundlegender Identitäts-Fehler, denn: Nichts in der Entwicklung des Menschen ist ,abgeschlossen', nicht einmal die Biologie des Menschen. Wir sitzen oder stehen nicht nur , auf den Schultern der (Erkenntnis-) Riesen', nutzen nicht nur die Straßentrassen, die die Römischen Heere vor 2000 Jahren durch Europa bauten als Topografie der Autobahnen. Wir nutzen Alphabet, Zahlen, Rechenweisen, Materialkenntnisse, Mechaniken und vieles mehr, allerdings auf unsere Weise. Und wir verändern unsere Körperlichkeit ebenso, wie wir massiv in jedwede Umwelt eingreifen. Nun will ich nicht in Geschichte, nicht in Ökologiediskurse ausweichen. 


\section{Die Frage ist:}

Wie beeinflusst das immer wieder neue Ringen um Abstraktion, Entwurf, Gestaltung unser kooperatives, kollaboratives, konflikthaftes, konsensfähiges Denken?

Wie entstehen Auswahlmuster, wie deren selektive Anwendung? Wie werden Machtgefüge hervorgebracht? Wie Konzepte und Realitäten von sozialer Gruppenorganisation, nennt man diese nun Ethnos, Demos, Plebs, Barbaren oder ,fremde Nachbarschaft'?

Und was genau benennen diese Wörter kategorial?

Das werde ich hier nicht auflösen können. Ich bleibe bei den Verhältnissen, die Menschen mit Dingen, Dingen mit Menschen haben und beziehe mich dabei auf die mediale Selbstbefähigung des Menschen (Faßler 1996), mediale Interaktion und auf den Forschungsrahmen, den ich beim Antritt der Professur an der Goethe-Universität Frankfurt umrissen habe: eine Anthropologie des Medialen.

Inzwischen ist es erforderlich geworden, die Anthropologie der Dinge, Abstraktionen und Medien auszuweiten in die Erforschung der Transfers von Wissen um Dinge, Organisation, Management, Ökologie, um nur weniges hier anzusprechen. Nicht nur der „Stammbaum des Homo sapiens (ist) kein übersichtliches und wohlgeordnetes Muster" (Maddox 2000: 298). Dies betrifft auch jede Handlungs-, Technologie- und Organisationsebene.

\section{Welt-Dinge und Ausbleichen von Kultur?}

Der jüngste Stand der Dinge ist: Sie, diese Dinge, sind keine Einzelwerke mehr, kein Personal Computing. Auch, wenn es ungewohnt zu lesen ist: Wir befinden uns in der Epoche eines "Social/Global/Total Computing", dem die Wörter und Sprachen abgerungen werden müssen, mit denen die Anschlussfähigkeiten von Ethnos, Demos, Selbstbestimmung und Selbstorganisation als Dimension eines technologischen Humanismus benannt und betrieben werden können.

Verharmlosen, verteufeln oder neutralisieren gilt nicht mehr. Auch gilt nicht mehr, die kulturelle und soziale Verfassung von IoT, Smartness-Konzepten, Kreativitäts-Modellen, Konferenz- und Erziehungsmodellen von ONLINE-Angeboten zu ignorieren. Einzelgeräte der PC-Generation sind nicht mehr die springenden Punkte, mit denen sich Wissenschaften befassen müssen. Lange schon geht es nicht mehr um PONG, dem Tennisspiel gegen oder mit Computer und Interface (was mich bei meinen Kneipenbesuchen der 1970er begleitete).

Alle Sozialverfassungen der Welt, die Digitalwirklichkeiten auf sich anwenden, verändern sich massiv. Und in ihnen werden

Subjekte zu User:innen,

Institutionen von datenmonopolen Netzwerken überlagert,

Soziales wird durch Plattform-Kapitale, durch „intangible capital“ (Haskel \& Westlake 2018) geformt,

Berufe auf dem Niveau von Prod-User:innen neu formuliert,

Demos zu einer Mischung aus First E Second Life, Biologie und "artificial life",

Kultur zu einer Kategorie der Miet-Kulturen von Google, Huawei, WhatsApp, Facebook, o.ä. 
Wohin das führt, kann niemand sagen.

Da aber jedes soziale Kommunikationsgeschehen von der datenökonomischen und datensozialen Transformation erfasst werden wird, sind Forschungskategorien erforderlich, mit denen die komplexen Dynamiken des Sozialen erfasst werden, - und in ,nahe Zukunft' verlängert werden können. Wissend, dass mehr als Vorschläge nicht daraus werden können und sollten. Zustände und Zukünfte sind zwar, wie es aus einer Richtung heißt, user-/consumerdriven, und aus der anderen Richtung: technology-driven.

Aber dieses ,treiben/steuern/fahren' ist weder in seiner Entstehung und Durchsetzung, noch in seinen möglichen Zielen dargestellt. Gerade die Diversität und Komplexität der Entwicklungen legen keinerlei Ziel fest. Sie ermöglichen keinerlei Eindeutigkeit, weder im hermeneutischen, noch im mathematischen Sinne. Diese menschengemachten Dynamiken sind ko-evolutionär ,ziellos', trotz ihrer augenblicklichen funktionalen Leistungsspitze. Wer freut sich nicht, wenn die Realität des Computer-Interfaces störungsfrei 24h am Tag in Echtzeit auf- und abgebaut wird?

Bislang liegen allerdings keine normativ orientierten Debatten um Daten- und DigitalKultur vor, keine Debatten über Daten-Völker, Daten-Migrant:innen, keine über die Machtspannungen zwischen "Gutenberg-Galaxis“, magischen Kanälen (McLuhan 1994) und Turing-Galaxis. Auch fehlen Entwicklungsmodelle, die auf einen technologischen Humanismus zielen oder auf eine humanisierte/urbanisierte/zivilisierte Datenökonomie.

Wir erleben ein Ausbleichen moderner Gesellschaftsformen (Faßler 2009; 2014), da die datenökonomischen Monopole keiner konkreten Gesellschafts- oder Kulturform dauerhaft bedürfen. Zugleich erleben wir eine Normen-Bleiche, da die für Normenentscheidung (bislang noch) erforderlichen Öffentlichkeiten kommunikativ und institutionell nicht zusammenkommen, derzeit.

Gerade auch innerhalb der Fächer Soziologie und Kulturanthropologie sind Bestrebungen erst am Anfang, dem Ausbleichen der (datenökonomischen und netztechnischen) Gesellschaftsform und der Normen-Bleiche entgegenzuwirken. Mit dem Ziel, genossenschaftliche Experimente von informations-ökonomischen Gemeinwohlbezügen zu unterstützen. Dies wird erfordern, sich einesteils von den Idealen überzeitlicher Sozialordnungen grundlegend zu trennen. Und es wird erfordern, nicht nur Rechte für Diversität und Differenzen einzufordern, sondern zugleich formulieren, worin das Gemeinwohl, die verbindlichen Zusammenhänge und die sozio-technologischen sowie ökologischen Grundlagen bestehen.

Dabei wird die bisherige Dreistufigkeit der Entwicklung von kybernetischer Informationsökonomie (= Digitalisierung) zu berücksichtigen sein. Die Entwicklungsverläufe von Datentechnologie, die weiter zu berücksichtigen sind, sind:

- $\quad$ additive Verläufe: Zusatz für Verwaltung und Steuerung durch Großrechenanlagen in Versicherungen, Banken, statischen Bereichen der Bauwirtschaft, Militär [Communication-Command-Control-/C3-Programme] (Entwicklungen seit den späten 1930ern, z. B. durch Z1/Z2 von Konrad Zuse, durch Enigma, Colossus seitens der USA und England in den 1940ern, sowie die Etablierung von Großrechenanlagen für die Unterstützung von unternehmerischen und politischen Verwaltungen seit den 1950ern),

- $\quad$ substitutive Verläufe: Seit 1956 forschungsstrategisch und industriell vorangetriebene Entwicklung von Artificial Intelligence (Dartmouth, 1. AI-Konferenz) als für Ersatz für körperlich-schwere Arbeiten (Robotik), sich wiederholende Fließband-Arbeit, komplexe Rechenverfahren in Versicherungen etc., und vor allem für die Konzepte selbstlernender Automatisierung, 
- und als soziale und globale Gesamtordnung, die durch die gegenwärtige Algorithmisierung von Hyperlinks dazu tendiert, ein automatisiertes Meta-Netzwerk des Sozialen zu bilden. Die Verknüpfung von lokalen Maschinenparks, globalen Vernetzungsprogrammen, individualisierenden Welt-Angeboten und kulturellen und sozialen Angeboten, die sich in Echtzeit bilden können und ebenso rasch verworfen werden können, überschreiten die früheren Angebote von additiv und substitutiv.

- Durch die global eingesetzten Algorithmen der Vernetzung und die globalen Märkte relativ einheitlich funktionierender Geräte - trotz der ökonomischen und sicherheitspolitischen Streits um Google, Facebook, Huawei etc.

- $\quad$ konstitutive Verläufe: Auf der Basis von sogenannter social networks, social technologies, social marketing, design, innovations, analytics, bonds, vorangetrieben durch PlattformKapitale und globale Datenindustrien seit Web 2.0 (also den 2000ern), entstehen Körperschaften und Sozialordnungen neuer Prägung. Sie werden von automatisierenden Programmen, Menschen, Maschinen, Algorithmisierung von Netzwerken getragen, die kein Territorium, keine kulturelle Gemeinschafts- und Konfliktentwicklung mehr teilen. Sie haben asymmetrische, sekündliche, gemietete Vernetzungen als Basis. Die globalen Datenökonomien, die derzeit durch The Big 9 (Webb 2019) vertreten sind, fügen Geschäftsbereiche zu Sozialverfassungen zusammen. Sie greifen nach der Grundlage von Legitimation, Legalisierung, Wissenskulturen (Faßler 2020). Sie eignen sich auch die politischen Bedingungen von Sozialen Verfassungen an, indem sie immer mehr soziale Gruppen integrieren, ihre Heterogenität in den Praxen datenkonformer Kommunikation umgehen.

Alle drei bisherigen Entwicklungsschwerpunkte angewandter Kybernetik (oder Digitalisierung) werden weiterhin gleichzeitig intensiviert werden.

Eine kulturanthropologisch begründete (Entstehungs- und) Veränderungsforschung sollte mit der Erforschung dieser Krisen der Sozial- und Politik-Verfassungen beginnen. Und dies nicht als Technologie-Kritik, sondern als Praxis-Kritik.

Zum Leitspruch dieser Erforschung generativer Realitäten könnte der Satz werden:

Die Praxis ist das Medium!

\section{Literatur}

Anderson, Chris (2008): The End of Theory: The Data Deluge Makes the Scientific Method

Obsolete. In: WIRED. https://www.wired.com/2008/06/pb-theory/. Letzter Zugriff: unbekannt.

Bateson, Gregory (1997 [1987]): Geist und Natur. Frankfurt am Main: Suhrkamp.

Bateson, Gregory (1996 [1985]): Ökologie des Geistes. Frankfurt am Main: Suhrkamp.

Boyer, Pascal (2004): Und Mensch schuf Gott. Stuttgart: Klett-Verlag.

Callon, Michel (2006): Can Methods for Analysing Large Numbers Organize a Productive

Dialogue with the Actors They Study? In: European Management Review 3/1, 7-16.

Couldry, Nick \& Henry Jenkins (2014): Participations: Dialogues on the Partcipatory Promise of Contemporary Culture and Politics. In: International Journal of Communication 8, 11071112.

Crouch, Colin (2015): Die bezifferte Welt. Wie die Logik der Finanzmärkte das Wissen bedroht. Berlin: Suhrkamp.

Dawkins, Richard (1976): The Selfish Gene. Oxford: Oxford University Press.

Dakwins, Richard (1982): The Extended Phenotype: The Long Reach of the Gene. Oxford:

Oxford University Press. 
Deschauer, Martin, Julian Meyer, Janine Seit \& Tina Wernicke (2009) (Hgs.): projekt:wissen. Von

Datenbergen, Informationsströmen und Wissensgenerierung. Kulturanthropologie Notizen Band 77, Frankfurt am Main.

Deschauer, Martin, Nora Geisler \& Lena Papasabbas (2014) (Hgs.): Black Box Brain. In:

Kulturanthropologie Notizen Band 82, Frankfurt am Main.

du Sautoy, Marcus (2019): The Creativity Code: How AI Is Learning to Write, Paint and Think.

London: 4th Estate.

du Sautoy, Marcus, Dan Segal \& Aner Shalev (2000): New Horizons in Pro-P Groups. Boston,

MA: Birkhäuser Boston.

Faßler, Manfred (1996): Mediale Interaktion. Speicher - Individualität - Öffentlichkeit.

München: W. Fink Verlag.

Faßler, Manfred (2009): Nach der Gesellschaft. Infogene Welten, anthropologische Zukünfte.

München: W. Fink Verlag.

Faßler, Manfred (2014): Das Soziale. Entstehung und Zukunft menschlicher Selbstorganisation.

München: W. Fink Verlag.

Faßler, Manfred (2020): Partizipation ohne Demokratie. Über Folgen der Netz- und Geopolitik von Facebook, Google, Amazon \& Co. Paderborn: Fink \& Schöningh/Brill.

Gapski, Harald \& Stephen Packard (Hgs.) (2021): Super-Scoring? Datengetriebene

Sozialtechnologien als neue Bildungsherausforderung. Düsseldorf, München: kopaed.

Haraway, Donna J. (1991): Simians, Cyborgs, and Women. The Reinvention of Nature. London: Routledge.

Heintz, Bettina (1993): Die Herrschaft der Regel. Zur Grundlagengeschichte des Computers.

Frankfurt am Main: Campus-Verlag.

Helmond, Anne (2013): The Algorithmization of the Hyperlink. In: Computational Culture 3/1, o.S.

Howard, Philip N. (2015): Pax Technica. How the Internet of Things May Set Us Free or Lock Us Up. Yale: Yale University Press.

Iser, Wolfgang (2013): Emergenz, Nachgelassene und verstreut publizierte Essays. Konstanz: Konstanz University Press.

Jaspers, Karl (1955 [1949]): Vom Ursprung und Ziel der Geschichte. Frankfurt am Main, Hamburg: Fischer Verlag.

Kelly, Kevin (1997): Das Ende der Kontrolle. Die biologische Wende in Wirtschaft, Technik und Gesellschaft. Mannheim: Bollmann.

Laboratory: Anthropology of Environment I Human Relations (Hg.) (2019): After Practice:

Thinking Through Matter(S) And Meaning Relationally. In: Berliner Blätter Band 80. Berlin: Panama Verlag. Volume I + II.

Leroi-Gourhan, André (1980): Hand und Wort. Die Evolution von Technik, Sprache und Kunst. Frankfurt am Main: Suhrkamp Verlag.

Löffler, David (2019): Generative Realitäten I. Weilerswist: Velbrück Wissenschaft.

Luhmann, Niklas (1984): Soziale Systeme. Grundriss einer allgemeinen Theorie. Frankfurt am Main: Suhrkamp Verlag.

Maddox, John (2000): Was zu entdecken bleibt. Über die Geheimnisse des Universums, den Ursprung des Lebens und die Zukunft der Menschheit. Frankfurt am Main: Suhrkamp Verlag.

Mainzer, Klaus (2007): Der Kreative Zufall. Wie das Neue in die Welt kommt. München: Beck. 
Marres, Noortje \& Carolin Gerlitz (2019): Social Media as Experiments in Sociality. In: Noortje Marres, Michael Guggenheim \& Alex Wilkie (Hgs.) Inventing the Social. Manchester: Mattering Press.

Maturana, Humberto R. \& Francisco J. Varela (2015 [1990]): Der Baum der Erkenntnis. Die biologischen Wurzeln des menschlichen Erkennens. Frankfurt am Main: Fischer Taschenbuch Verlag.

Mayer-Schönberger, Viktor \& Kenneth Cukier (2013): Big Data. Die Revolution, die unser Leben verändern wird. München: Redline.

McLuhan, Herbert M. (1994): Die magischen Kanäle. Understanding Media. Dresden: Verlag der Kunst.

Milan, Stefania (2015): When Algorithms Shape Collective Action: Social Media and the Dynamics of Cloud Protesting. In: Social Media + Society 1/2, 1-10.

Moscovici, Serge (1990): Versuch über die menschliche Geschichte der Natur. Frankfurt am Main: Suhrkamp Verlag.

Mühlmann, Heiner (2011): Die Natur der Kulturen. Entwurf einer kulturgenetischen Theorie. Paderborn: Fink.

Peckhaus, Volker (2005): Oskar Becker und die Philosophie der Mathematik. München: Fink. Polanyi, Karl (1979): Ökonomie und Gesellschaft. Frankfurt am Main: Suhrkamp Verlag.

Polanyi, Karl 2017 [1973]: The Great Transformation. Politische und ökonomische Ursprünge von Gesellschaften und Wirtschaftssystemen. Berlin: Suhrkamp Verlag.

Popitz, Heinrich (1992): Phänomene der Macht. Autorität - Herrschaft - Gewalt - Technik. Tübingen: Mohr Siebeck.

Rheingold, Howard (1992): Virtuelle Welten. Reisen im Cyberspace. Reinbek bei Hamburg: Rowohlt.

Riedl, Rupert (2000): Strukturen der Komplexität. Eine Morphologie des Erkennens und Erklärens. Berlin: Springer.

Riedl, Rupert (2003): Riedls Kulturgeschichte der Evolutionstheorie. Die Helden, ihre Irrungen und Einsichten. Berlin: Springer.

Rorty, Richard (1992): Kontingenz, Ironie und Solidarität. Frankfurt am Main: Suhrkamp Verlag.

Sassen, Saskia (2015): Ausgrenzungen. Brutalität und Komplexität in der globalen Wirtschaft, Frankfurt am Main: Fischer Verlag.

Schmidt, Eric \& Jared Cohen (2013): The New Digital Age. Reshaping the Future of People, Nations and Business. New York: Knopf.

Schurz, Gerhard (2011): Evolution in Natur und Kultur. Eine Einführung in die verallgemeinerte Evolutionstheorie. Heidelberg: Springer Verlag.

Schwab, Klaus (2019): Die Zukunft der Vierten Industriellen Revolution. Wie wir den digitalen Wandel gemeinsam gestalten. München: Deutsche Verlags-Anstalt.

Seife, Charles (2000): Zero. The Biography of a Dangerous Idea. New York: Penguin Books.

Triebel, Claas (2013): Fünf Jahre nach dem Ende der Theorie. In: Heise.de. https://www.heise.de/tp/features/Fuenf-Jahre-nach-dem-Ende-der-Theorie-3399508.html. Letzter Zugriff: unbekannt.

van Dijk, Jose (2013): The Culture of Connectivity: A Critical History of Social Media. Oxford: Oxford University Press.

Vester, Frederic (2019): Die Kunst vernetzt zu denken. Ideen und Werkzeuge für einen neuen Umgang mit Komplexität; ein Bericht an den Club of Rome. München: Pantheon 
von Foerster, Heinz (2008): Der Anfang von Himmel und Erde hat keinen Namen. Eine Selbsterschaffung in sieben Tagen. Berlin: Kulturverlag Kadmos.

Webb, Amy (2019): The Big Nine. How the Tech Titans and Their Thinking Machines Could Warp Humanity. New York: PublicAffairs.

Wieser, Wolfgang (2007): Gehirn und Genom. Ein neues Drehbuch für die Evolution. München: Beck.

Wilson, Edward O. (2013): Die Einheit des Wissens. München: Goldmann.

\section{Weitere Literatur ${ }^{4}$}

Aigner, Josef Christian, Theo Hug, Martina Schuegraf \& Angela Tilllman (2015): Medialisierung und Sexualisierung. Wiesbaden: Springer Verlag.

Amos, Martyn (2006): Genesis Machines. The New Science of Biocomputing. London: Overlook. Asdal, Kristin (2008): Enacting Things Through Numbers: Taking Nature into Account/ing. In: Geoforum 39, 123-132.

Baecker, Dirk (2016): Wozu Theorie? Berlin: Suhrkamp Verlag.

Dawkins, Richard (2010): Der erweiterte Phänotyp. Der lange Arm der Gene. Wiesbaden: Springer.

Diamond, Jared (2005): Kollaps. Warum Gesellschaften überleben oder untergehen. Frankfurt am Main: Fischer.

Drucker, Peter F. (1993): The Age of Social Transformation. In: The Atlantic Monthly 11, 53-80.

Duerr, Hans Peter (1984): Sedna oder Die Liebe zum Leben. Frankfurt am Main: Suhrkamp Verlag.

Faßler, Manfred (2005): Erdachte Welten. Die mediale Evolution globaler Kulturen. Wien, New York: Springer.

Faßler, Manfred (2008): Der infogene Mensch. Entwurf einer Anthropologie. München: W. Fink Verlag.

Faßler, Manfred (2012): Kampf der Habitate. Neuerfindung des Lebens im 21. Jahrhundert. Wien, New York: Springer.

Fidler, Roger (1997): Mediamorphosis. Understanding New Media, London: SAGE Publications.

Gentzel, Peter, Friedrich Krotz, Jeffrey Wimmer \& Rainer Winter (2019): Das vergessene Subjekt. Subjektkonstitutionen in mediatisierten Alltagswelten. Wiesbaden: Springer.

Gummert, Henrik, Jelena Henkel-Otto \& Dirk H. Medebach (2017): Medien und Kulturen des Konflikts. Wiesbaden: Springer.

Haidle, Noël Haidle (2010): Working-Memory Capacity and the Evolution of Modern Cognitive Potential Implications from Animal and Early Human Tool Use. In: Current Anthropology 51/1, 149-166.

Haskel, Jonathan \& Stian Westlake (2018): Capitalism without Capital. The Rise of the Intangible Economy. Princeton: Princeton University Press.

Jörissen, Benjamin \& Torsten Meyer (2015): Subjekt Medium Bildung. Wiesbaden: Springer Verlag.

Johnson, Steven (2006): Neue Intelligenz. Warum wir durch Computerspiele und TV klüger werden. Köln: Kiepenheuer \& Witsch.

\footnotetext{
${ }^{4}$ Wir haben uns entschieden, Literatur, die Manfred Faßler im Literaturverzeichnis angegeben, aber nicht im Text zitiert hat, beizubehalten und hier in einem gesonderten Verzeichnis aufzulisten.
} 
Laughlin, Robert B. (2008): Das Verbrechen der Vernunft. Betrug an der Wissensgesellschaft. Frankfurt am Main: Suhrkamp Verlag.

Laughlin, Robert B. (2007): Abschied von der Weltformel. München: Piper.

Levitsky, Stevens \& Daniel Ziblatt (2018): How Democracies Die. New York: Penguin.

Lumsden, Charles J. \& Edward O. Wilson (2005 [1981]): Genes, Mind, and Culture. The

Coevolutionary Process. Singapur: World Scientific Pub. Co.

Ochs, Carsten, Michael Friedewald, Thomas Hess \& Jörn Lamla (2019): Die Zukunft der

Datenökonomie. Zwischen Geschäftsmodell, Kollektivgut und Verbraucher-

schutz.Wiesbaden: Springer Verlag.

Rifkin, Jeremy (2000): Access. Das Verschwinden des Eigentums. Warum wir weniger besitzen und mehr ausgeben werden. Frankfurt am Main, New York: Campus-Verlag.

Wolfgang Sützl, Felix Stalder, Ronald Maier \& Theo Hug (2012): Media, Knowledge and Education: Cultures and Ethics of Sharing. Innsbruck: Innsbruck University Press.

Tomasello, Michael (2010): Die Ursprünge der menschlichen Kommunikation. Frankfurt am Main: Suhrkamp Verlag.

Tomasello, Michael (2014): Eine Naturgeschichte des menschlichen Denkens. Berlin: Suhrkamp Verlag.

van der Nagel, Emily (2018): 'Networks that work too well': Intervening in Algorithmic Connections. In: Media International Australia 168/1, 81-92.

van Dijk, José, David Nieborg \& Thomas Poell (2019): Reframing Platform Power. In: Internet Policy Review 8/2, o. S.

von Glaserfeld, Ernst (1997): Wege des Wissens. Konstruktivistische Erkundungen durch unser Denken. Heidelberg: Carl-Auer-Systeme.

Winter, Raimer (2014): Widerstand im Netz. Zur Herausbildung einer transnationalen Öffentlichkeit durch netzbasierte Kommunikation. Bielefeld: Transcript.

Crouch, Colin (2015): Die bezifferte Welt. Wie die Logik der Finanzmärkte das Wissen bedroht, Berlin: Suhrkamp Verlag.

\section{Autoreninformation}

Manfred Faßler war habilitierter Soziologe (Freie Universität Berlin). Nach seiner Promotion (1979) lehrte er an der FU / FHW / EFH in Berlin (W). Bereits in Berlin Anfang der 1980er und später in Verbindung mit einem USA-Aufenthalt befasste er sich mit Computertechnologie als Soziale Formation. 1994 legte er seine Habilitationsschrift "Mediale Interaktion" vor. Zeitgleich mit der Erstellung der Habilitation war er Studienleiter und Leiter des Begabtenförderungswerkes Evangelisches Studienwerk e.V. 1995 wurde er auf die Lehrkanzel für Kommunikationstheorie an der Universität für angewandte Kunst Wien berufen; 1997 wurde er dort Abteilungsleiter (Dekan) für den Bereich „Visuelle Mediengestaltung“; im September 2000 wechselte er an die Goethe Universität Frankfurt. In zahlreichen Büchern, Konferenz- und Buchbeiträgen entwickelte er den Ansatz einer „Anthropologie des Medialen“. Über Gastprofessuren an der Universität für angewandte Kunst Wien, Universität Basel, Alpen-Adria-Universität Klagenfurt, Goldsmiths College London sowie Universidade de Sao Paolo entstand ein internationales Forschungsnetzwerk Anthropologie des Medialen/FAME. 